\title{
Invasive Paget's disease of the vulva treated with a combination of surgery and concurrent chemoradiotherapy: A case report
}

\author{
YOSHIHIDE INAYAMA ${ }^{1,2}$, KAORU ABIKO $^{1}$, TAITO MIYAMOTO ${ }^{1}$, AKIHITO HORIE $^{1}$, KEN YAMAGUCHI $^{3}$, \\ TSUKASA BABA $^{1}$, NORIOMI MATSUMURA ${ }^{4}$, SACHIKO MINAMIGUCHI $^{5}$ and MASAKI MANDAI ${ }^{1}$ \\ ${ }^{1}$ Department of Gynecology and Obstetrics, Kyoto University Graduate School of Medicine, Kyoto, \\ Kyoto 606-8507; ${ }^{2}$ Department of Obstetrics and Gynecology, Toyooka Hospital, Toyooka, Hyogo 668-8501; \\ ${ }^{3}$ Department of Obstetrics and Gynecology, National Hospital Organization Kyoto Medical Center, Kyoto, Kyoto 612-8555; \\ ${ }^{4}$ Department of Obstetrics and Gynecology, Kinki University Faculty of Medicine, Osaka-Sayama, Osaka 589-8511; \\ ${ }^{5}$ Department of Diagnostic Pathology, Kyoto University Graduate School of Medicine, Kyoto, Kyoto 606-8507, Japan
}

Received July 16, 2018; Accepted September 17, 2018

DOI: $10.3892 / \mathrm{mco} .2018 .1719$

\begin{abstract}
Invasive Paget's disease of the vulva (IP) is rare among patients with vulvar cancer. Radiation therapy and chemotherapy are not considered as radical, whereas surgical resection of the tumor with abdominal lymphadenectomy is highly invasive. Thus, more effective and less invasive treatments for IP are required. The present study reports a case of a 64-year-old woman with IP, who was treated with a combination of surgery and concurrent chemoradiotherapy (CCRT). The patient was diagnosed with IP with suspected lymph node metastases to the inguinal and pelvic lymph nodes, after having suffered from pruritus vulvae for 7 years. Following mapping biopsy, wide local excision, bilateral inguinal lymph node resection and laparoscopic pelvic lymphadenectomy were successfully performed. The vulva was reconstructed with a local fat flap. Postoperative pathological examination revealed metastases to the bilateral superficial inguinal and the left obturator and lateral suprainguinal lymph nodes. Adjuvant CCRT (whole pelvic irradiation, 50.4 Gy with weekly cisplatin, $40 \mathrm{mg} / \mathrm{m}^{2}$ ) was completed without notable complications. Therefore, laparoscopic pelvic lymphadenectomy may be useful in determining the irradiation field for adjuvant CCRT in patients with advanced IP.
\end{abstract}

Correspondence to: Dr Kaoru Abiko, Department of Gynecology and Obstetrics, Kyoto University Graduate School of Medicine, 54 Shogoin Kawahara-cho, Sakyo-ku, Kyoto, Kyoto 606-8507, Japan E-mail:kaoruvc@kuhp.kyoto-u.ac.jp

Abbreviations: CCRT, concurrent chemoradiotherapy; IEP, intraepithelial Paget's disease; IP, invasive Paget's disease of the vulva

Key words: concurrent chemoradiotherapy, invasive Paget's disease, laparoscopic pelvic lymphadenectomy, staging surgery, vulvar cancer

\section{Introduction}

Invasive Paget's disease of the vulva (IP) is a rare entity among patients with vulvar cancer, accounting for $<1 \%$ of all vulvar malignancies (1). Radiation therapy and chemotherapy are not considered to be radical, whereas surgical resection of the tumor with abdominal lymphadenectomy is highly invasive $(2,3)$. Paget's disease of the vulva is classified into primary and secondary, which may be distinguished using immunohistochemistry (3-5). Primary Paget's disease is subdivided into three categories, namely intraepithelial Paget's disease (IEP), IP, and Paget's disease as a manifestation of an underlying adenocarcinoma of a skin appendage or a vulvar gland (4-6). While IEP is usually treated with surgery and its prognosis is relatively good, IP is associated with a poor prognosis $(1,3)$ and a treatment strategy for advanced IP has not yet been standardized $(2,6)$. Thus, more effective and less invasive treatments for IP are required.

We encountered a case of IP with metastases to the pelvic lymph nodes, which was effectively treated with a combination of wide local excision, inguinal lymph node resection and laparoscopic pelvic lymphadenectomy, followed by concurrent chemoradiotherapy (CCRT).

\section{Case report}

A 64-year-old woman visited Kyoto University Hospital with a rapidly growing mass in the vulva accompanied by pruritus. The patient had first experienced pruritus in the vulva 7 years prior and visited a local hospital. A topical cream was prescribed, but the pruritus persisted. Finally, the patient found a growing mass in the vulva and visited the hospital again. A wide erythematous rash and a hemorrhagic red mass were observed in the vulva and the patient was diagnosed with vaginal cancer and was referred to our hospital for further evaluation. At presentation, a solid mass measuring $4.3 \mathrm{~cm}$ was observed in the right vulva, with bilateral inguinal lymphadenopathy (Fig. 1A). A biopsy of the mass revealed invasive cancer cells. The results of the immunohistochemical examination revealed that the tumor cells were positive for 

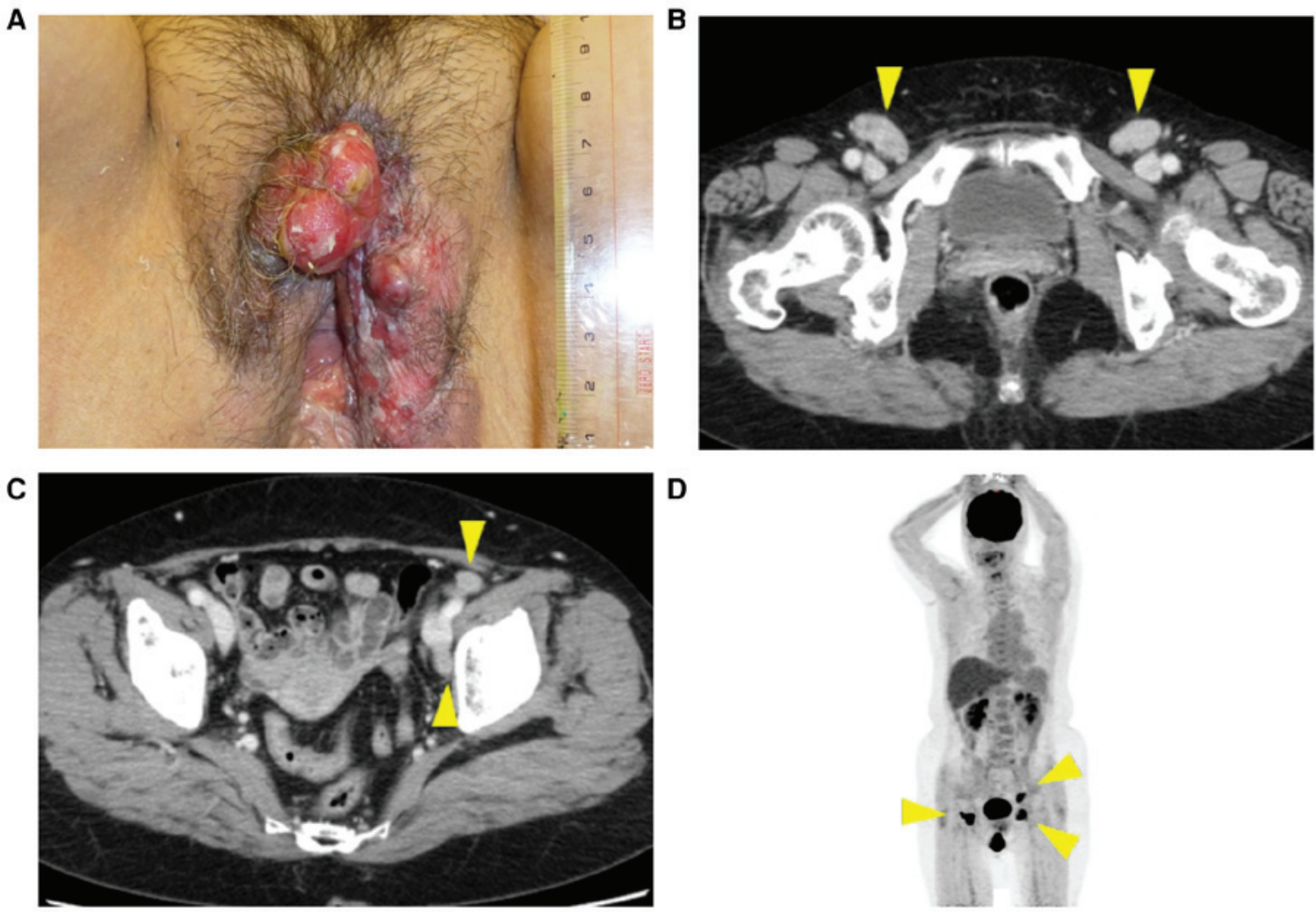

Figure 1. Preoperative findings of invasive Paget's disease of the vulva. (A) Macroscopic appearance of the vulvar tumor. A red mass measuring 4.3x3.6 cm near the clitoris and a widespread erythematous rash in the vulva were observed. (B) Enlarged bilateral superficial inguinal lymph nodes (arrowheads) were observed on computed tomography scan. (C) Enlarged left obturator and lateral supra-inguinal lymph nodes (arrowheads) were visualized using computed tomography. (D) On positron emission tomography, fluorodeoxyglucose uptake (arrowheads) was observed in the left obturator and lateral supra-inguinal lymph nodes, as well as the vulvar tumor.

cytokeratin (CK)7 and negative for p63 and CK20. Biopsies from the erythematous rash surrounding the mass revealed Paget's cells in the epithelium, indicating that the invasive tumor cells originated from primary vulvar Paget's cells, and the case was diagnosed as IP. Bilateral superficial inguinal lymphadenopathy and left obturator and lateral suprainguinal lymphadenopathy were observed on computed tomography, magnetic resonance imaging and positron emission tomography examination (Fig. 1B-D). After mapping biopsy, local tumor resection, bilateral inguinal lymph node resection and laparoscopic pelvic lymphadenectomy were performed, followed by adjuvant CCRT. The vulva was reconstructed using a local fat flap (Fig. 2A), and laparoscopic pelvic lymphadenectomy was performed to determine the irradiation field for CCRT (Fig. 2B). The operative time was $8 \mathrm{~h}$ and $17 \mathrm{~min}$ and the total intraoperative blood loss was $66 \mathrm{~g}$. Postoperative pathological examination revealed invasive adenocarcinoma arising from non-invasive Paget's disease (Fig. 3). Metastases to the bilateral superficial inguinal nodes and left obturator and lateral suprainguinal nodes were identified. CCRT (whole pelvic irradiation, $50.4 \mathrm{~Gy} / 28$ fractions with weekly cisplatin 7 cycles, $40 \mathrm{mg} / \mathrm{m}^{2}$ ) was completed without notable complications. The patient experienced an incomplete fracture of the left femoral head, which was possibly treatment-related, 12 months after the treatment and was treated conservatively with the administration of non-steroidal anti-inflammatory drugs and rest. The patient remained alive without recurrence 15 months after the treatment.

\section{Discussion}

Paget's disease of the vulva is classified into primary and secondary Paget's disease; the former is of cutaneous origin, whereas the latter originates from other malignancies, such as those from the urogenital or gastrointestinal tracts (3-5). Primary Paget's disease is subdivided into three categories: Intraepithelial Paget's disease (IEP), IP, and Paget's disease as a manifestation of an underlying adenocarcinoma of a skin appendage or vulvar gland (4-6). Immunohistochemistry is useful for the differential diagnosis of primary and secondary Paget's disease; Paget's cells of vulvar origin express CK7 and carcinoembryonic antigen, whereas those secondary to urothelial cancer are positive for CK20, p63, uroplakin-III and GATA-3, and those secondary to anorectal adenocarcinoma are positive for CK20, CDX2 and MUC2, but negative for CK7 (3,7). The tumor cells in the present case were positive for CK7 and negative for p63 and CK20, suggesting a vulvar origin.

While IEP is usually treated with surgery and its prognosis is relatively good, the prognosis of IP is poor $(1,3)$ and treatment strategies for advanced IP have not yet been standardized $(2,6)$. IP is usually treated as squamous cell carcinoma (SCC) of the vulva (3). Stage IV SCC of the vulva is usually treated with chemotherapy or CCRT (8-10). When Paget's disease is treated with radiotherapy, a radiation dose of 40-50 Gy is recommended for IEP and 55-65 Gy for IP $(3,11,12)$. A previous case report described the efficacy of CCRT in advanced IP; 
A

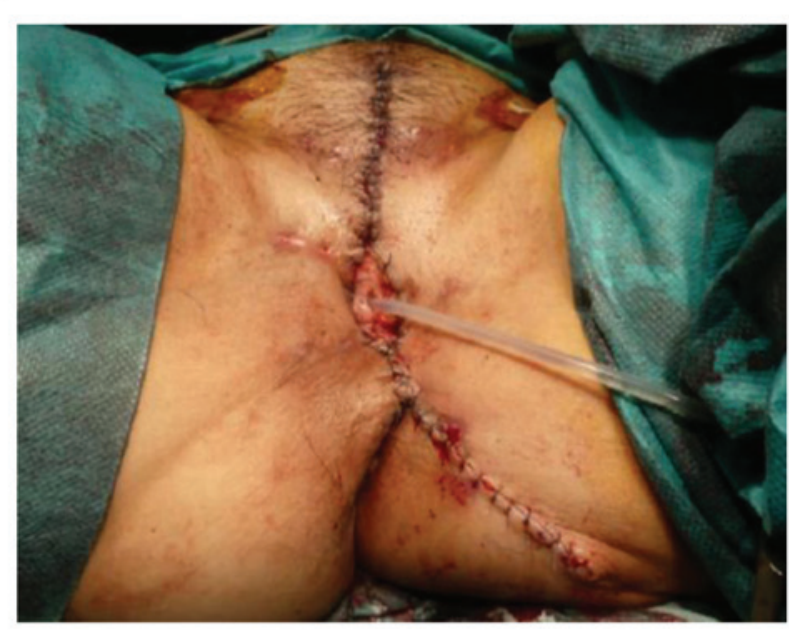

B Left external iliac vein Left obturator node

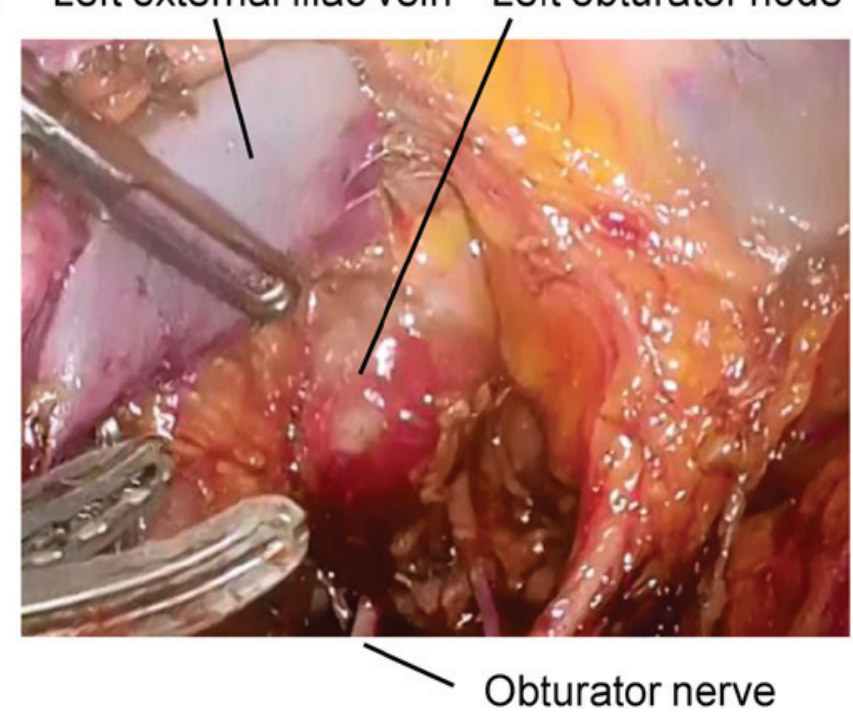

Figure 2. Intraoperative findings of the invasive Paget's disease of the vulva. (A) After local resection of the tumor, the vulva was reconstructed with a local fat flap. (B) Laparoscopic pelvic lymphadenectomy; an enlarged left obturator lymph node is indicated.
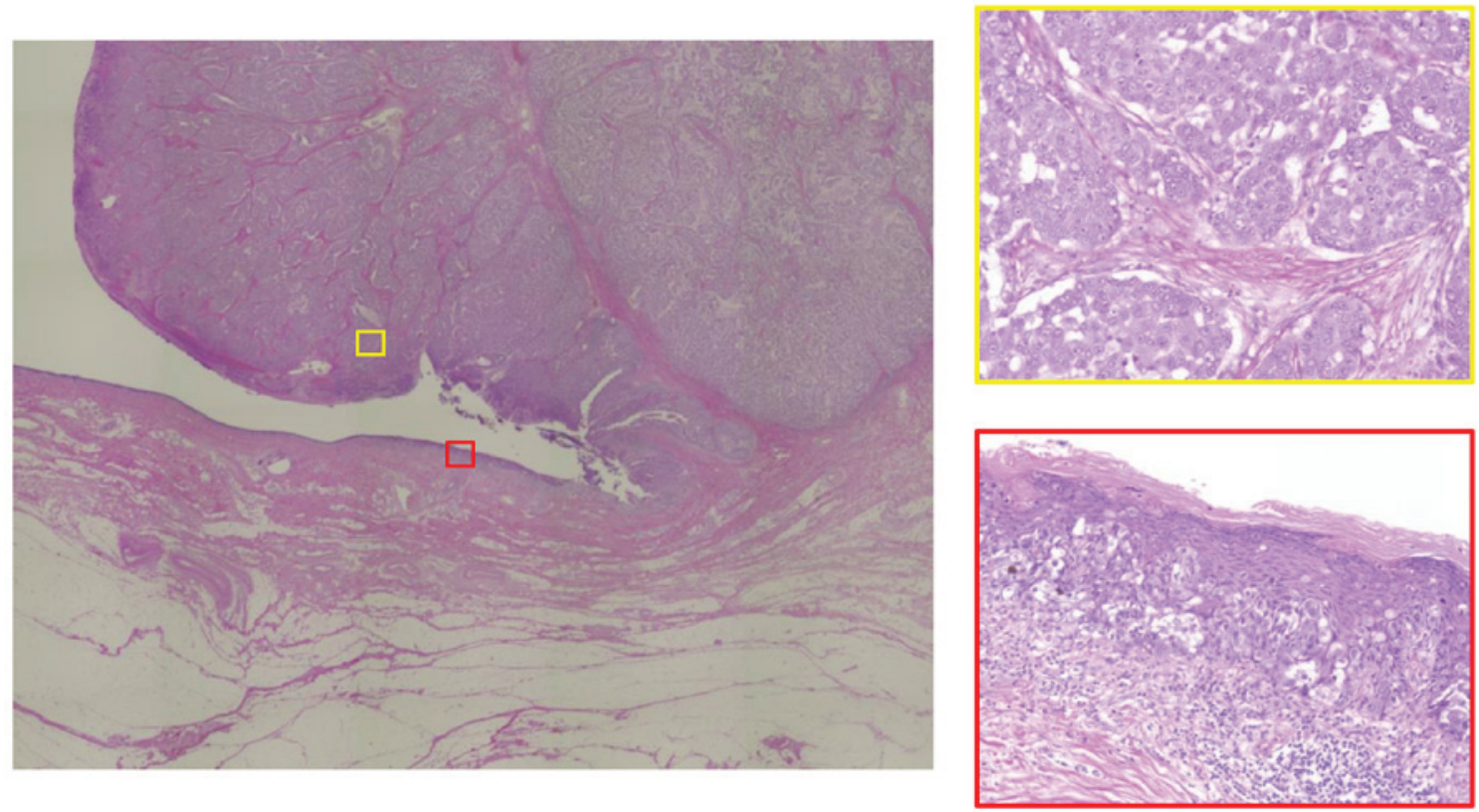

Figure 3. Hematoxylin and eosin staining of the resected specimen of the invasive Paget's disease of the vulva. Note the protrusion of invasive adenocarcinoma (yellow square) abruptly arising on a background of non-invasive Paget's disease (red square). Left, loupe image; magnification, $\sim \mathrm{x} 5$. Upper-right; magnification, x200. Lower-right; magnification, x200.

good disease control was obtained within the irradiation field, although the metastatic lesions outside the irradiation field progressed (2). In the present case, metastases were limited to inguinal and pelvic lymph nodes, both of which were within the field of whole pelvic irradiation. Thus, it was suggested that the disease may be controlled with CCRT.

Historically, radical vulvectomy was previously performed for vulvar SCC, but this procedure is extremely invasive and severe complications, such as postoperative wound dehiscence, infection, lymphedema and psychosexual impairment, are common $(8,13)$. Moreover, recurrence following radical vulvectomy is not rare in Paget's disease (6).
Wide local resection, which is a less invasive approach, is currently becoming the standard treatment modality for early-stage SCC of the vulva and primary $\operatorname{IEP}(6,14)$. Our patient did not experience wound dehiscence or infection following wide local resection, and underwent adjuvant CCRT immediately after the surgery. However, it is difficult to obtain clear surgical margins in Paget's disease, as Paget's cells spread through the epidermis $(15,16)$. Intraoperative evaluation of the surgical margins is conducted in some cases, but it is associated with $\sim 10 \%$ false negative outcomes, since Paget's cells are difficult to evaluate on frozen sections $(3,17)$. Therefore, in the present case, mapping biopsy was conducted 
prior to surgery, as it has been reported to be useful for determining the surgical margins in extramammary Paget's disease (16).

Although surgery alone does not appear to be sufficient for the treatment of IP (15), surgery as a staging procedure may be useful, as accurate staging is key to determining treatment strategy for IP. It has been reported that over one-third of patients with Paget's disease with metastases to the lymph nodes succumb to the disease (1), which suggests that treating lymph node metastases is crucial. Laparoscopic pelvic lymphadenectomy is widely performed in gynecological malignancies, such as cancers of the uterine corpus and cervix; this procedure is safe and less invasive, with a shorter hospital stay compared with laparotomy (18). Laparoscopic lymphadenectomy is conducted to determine the irradiation field in cervical cancer and in some cases of vulvar SCC $(18,19)$; hence, it may also be useful for IP with suspected lymph node metastases. Bilateral inguinal lymphadenectomy was avoided, as lymphadenectomy combined with radiotherapy may cause severe lymphedema (14). Instead, resection of the enlarged lymph nodes was performed, and the patient did not develop lymphedema after CCRT.

In conclusion, we herein present a case of invasive Paget's disease treated successfully without severe complications using a combination of local excision and laparoscopic pelvic lymphadenectomy as a staging surgery followed by adjuvant CCRT. Laparoscopic pelvic lymphadenectomy may be useful for determining the irradiation field for adjuvant CCRT in cases with advanced IP. However, further studies are required to establish an optimized treatment strategy for IP.

\section{Acknowledgements}

Not applicable.

\section{Funding}

No specific grant was received.

\section{Availability of data and materials}

Not applicable.

\section{Authors' contributions}

YI and KA treated the patient and wrote the paper. TM, AH, KY, TB and NM treated the patient and edited the manuscript. SM made the pathological diagnosis and edited the manuscript. MM approved the final version of the manuscript for publication. All authors agree with the content of the manuscript submitted for publication.

\section{Ethics approval and consent to participate}

Not applicable.

\section{Patient consent for publication}

Written informed consent was obtained from the patient for publication of the case details and associated images.

\section{Competing interests}

The authors declare that they have no competing interests.

\section{References}

1. Parker LP, Parker JR, Bodurka-Bevers D, Deavers M, Bevers MW, Shen-Gunther J and Gershenson DM: Paget's disease of the vulva: Pathology, pattern of involvement, and prognosis. Gynecol Oncol 77: 183-189, 2000.

2. Yamamoto R, Sakuragi N, Shirato H, Shimizu M and Fujimoto S: Radiotherapy with concurrent chemotherapy for vulvar adenocarcinoma associated with extramammary Paget's disease. Gynecol Oncol 80: 267-271, 2001.

3. van der Linden M, Meeuwis KA, Bulten J, Bosse T, van Poelgeest MI and de Hullu JA: Paget disease of the vulva. Crit Rev Oncol Hematol 101: 60-74, 2016.

4. Wilkinson EJ and Brown HM: Vulvar Paget disease of urothelial origin: A report of three cases and a proposed classification of vulvar Paget disease. Hum Pathol 33: 549-554, 2002.

5. Kurman RJ, Ellenson LH and Ronnett BM (eds.): Blaustein's Pathology of the Female Genital Tract 6th edition. Springer+Business Media, New York, pp81-86, 2011.

6. Delport ES: Extramammary Paget's disease of the vulva: An annotated review of the current literature. Australas J Dermatol 54: 9-21, 2013.

7. Kurman RJ, Carcangiu ML, Herrington CS and Young RH (eds.): World Health Organization Classification of Tumours. Int Agency Res Cancer, Lyon Cedex 08, pp236-237, 2014.

8. Deppe G, Mert I and Winer IS: Management of squamous cell vulvar cancer: A review. J Obstet Gynaecol Res 40: 1217-1225, 2014.

9. Mak RH, Halasz LM, Tanaka CK, Ancukiewicz M, Schultz DJ, Russell AH and Viswanathan AN: Outcomes after radiation therapy with concurrent weekly platinum-based chemotherapy or every-3-4-week 5-fluorouracil-containing regimens for squamous cell carcinoma of the vulva. Gynecol Oncol 120: 101-107, 2011.

10. Moore DH, Ali S, Koh WJ, Michael H, Barnes MN, McCourt CK, Homesley HD and Walker JL: A phase II trial of radiation therapy and weekly cisplatin chemotherapy for the treatment of locally-advanced squamous cell carcinoma of the vulva: A gynecologic oncology group study. Gynecol Oncol 124: 529-533, 2012.

11. Besa P, Rich TA, Delclos L, Edwards CL, Ota DM and Wharton JT: Extramammary Paget's disease of the perineal skin: Role of radiotherapy. Int J Radiat Oncol Biol Phys 24: 73-78, 1992.

12. Hata M, Omura M, Koike I, Wada H, Miyagi E, Tayama Y, Odagiri K, Minagawa Y, Ogino I and Inoue T: Role of radiotherapy as curative treatment of extramammary Paget's disease. Int J Radiat Oncol Biol Phys 80: 47-54, 2011.

13. De Hullu JA, Hollema H, Lolkema S, Boezen M, Boonstra H, Burger MP, Aalders JG, Mourits MJ and Van Der Zee AG: Vulvar carcinoma. The price of less radical surgery. Cancer 95: 2331-2338, 2002.

14. Hacker NF, Eifel PJ and van der Velden J: Cancer of the vulva. Int J Gynecol Obstet 119 (Suppl 2): S90-S96, 2012.

15. Kodama S, Kaneko T, Saito M, Yoshiya N, Honma S and Tanaka K: A clinicopathologic study of 30 patients with Paget's disease of the vulva. Gynecol Oncol 56: 63-70, 1995.

16. Kato T, Fujimoto N, Fujii N and Tanaka T: Mapping biopsy with punch biopsies to determine surgical margin in extramammary Paget's disease. J Dermatol 40: 968-972, 2013.

17. Zhu Y, Ye DW, Chen ZW, Zhang SL and Qin XJ: Frozen section-guided wide local excision in the treatment of penoscrotal extramammary Paget's disease. BJU Int 100: 1282-1287, 2007

18. Walker JL, Piedmonte MR, Spirtos NM, Eisenkop SM, Schlaerth JB, Mannel RS, Spiegel G, Barakat R, Pearl ML and Sharma SK: Laparoscopy compared with laparotomy for comprehensive surgical staging of uterine cancer: Gynecologic Oncology Group Study LAP2. J Clin Oncol 27: 5331-5336, 2009.

19. Klemm P, Marnitz S, Köhler C, Braig U and Schneider A: Clinical implication of laparoscopic pelvic lymphadenectomy in patients with vulvar cancer and positive groin nodes. Gynecol Oncol 99: 101-105, 2005. 